\title{
A New Asymmetric Fully Homomorphic Encryption Scheme for Cloud Banking Data
}

\author{
Zana Thalage Omar \\ Department of Computer \\ College of Science and Technology \\ University of Human Development, \\ Sulaimani, Iraq \\ zana.omar@uhd.edu.iq
}

\author{
Fadhil Salman Abed \\ Department of Information Technology \\ Kalar Technical Institute \\ Sulaimani Polytechnic University \\ Kalar,Iraq \\ fadhil.abed@spu.edu.iq
}

Shaimaa Khamees Ahmed

Computer Engineering

College of Engineering

University of Diyala Diyala, Iraq

shaymaakhamees88@gmail.com

\section{Article Info \\ Volume 5 - Issue 2 - \\ December 2020 \\ DOI: \\ 10.24017/science.2020.2.12 \\ Article history: \\ Received: 24 November 2020 \\ Accepted: 24 December 2020}

\section{Keywords:}

Fully Homomorphic

Encryption, Cloud computing,

Asymmetric Encryption, Large number, Banking security.

\begin{abstract}
Most banks in our time still use the common traditional systems of high cost and relatively slow, we are now in the era of speed and technology, and these systems do not keep pace with our current age, so saving cost and time will be considered a fantastic thing for banks. The way to that is to implement cloud computing strategies with Considering data security and protection when it comes to using the cloud. The best solution to protect data security on the cloud is fully homomorphic encryption systems. The time it takes to encrypt and decrypt data is one of the main barriers it faces. Our current research provides a new algorithm for a publicly-keyed encryption system to keep bank data from tampering and theft when stored on the cloud computing platform, and our new system achieves fully Homomorphic Encryption, which allows mathematical operations to be performed on the encrypted text without the need for the original text. The security of the new system depends on the issue of analyzing huge integers, which reach 2048 bits, to their prime factors, which are considered almost impossible or unsolvable. A banking application has also been created that encrypts the data and then stores it on the cloud. The application allows the user to create accounts and deposits, transfer and withdraw funds, and everything related to banking matters.
\end{abstract}




\section{INTRODUCTION}

The world is witnessing rapid development and prosperity of cloud computing, as cloud computing allows the sharing of services such as (applications, storage, processing) with cloud users. The focus is on increasing the effectiveness of shared resources[1]. One of the services provided by the cloud is to save users 'data on the cloud, hence the challenges and difficulties facing the cloud providers begin, as it is their responsibility to protect the security of user data on the one hand, On the other hand, the user does not fully trust the cloud providers because they can access, modify, and delete user data Intentionally, this issue is an obstacle to cloud providers[2]. Another phenomenon is a problem when storing data on the cloud as data exchange has become a common phenomenon among cloud providers under the service agreement because this phenomenon occurs in the scenes where the data owner is not aware of this process and is considered a violation of privacy Security of user data, especially untrusted parties may participate in this process [3]. Many believe that the solution lies in the use of encryption methods when storing data in the cloud and certainly should not use low-level security encryption methods. On the contrary, it must use high-level encryption methods in terms of security[4]. Most of the existing encryption systems face two main challenges. The first is that The principal distributions face threats in most symmetric key encryption systems [5]. The second is that data must be decrypted to make adjustments to it. Therefore, cloud providers have the decryption key, and thus the data becomes unsafe[6]. In this paper, we focus on the second challenge, where we create an encryption algorithm that allows modifications to the encrypted data without the need to decrypt it. This type of encryption is called Homomorphic Encryption Systems (HE)[7]. The term "homomorphism" is derived from a Greek word-initially composed of two parts. "Homos," which means the same, and "Morphic" means the form, this type of encryption (HE) is used in computer science, where it can convert plain text into encrypted text and make adjustments to it without the need to decrypt it[7]. This type of encryption is done through three stages: the stage of generation of the encryption key, the stage of encryption, and the stage of decryption. There are several types of it, one that supports multiplication operations, one that supports addition operations, and these two types are called (Partial Homomorphic Encryption) [8], [9]. And one that supports multiplication and addition operations together and is called (Fully Homomorphic Encryption), which is the type that We present in this paper. It supports addition and multiplication operations on encrypted data without the need to decrypt the data. The proposed algorithm generates the encryption key as described in Section 8, the key generation part, and then the data is encrypted using the encryption key through a mathematical algorithm described in the encryption part in Section 8 . The data is stored on the cloud in an encrypted form and when any modification or addition is made to the data, Amendment to it while it is in its encrypted state without the need to decrypt it, as the decryption key is owned by the owner of the data only and can decrypt the data through the mathematical equation described in the decryption part in Section 8 Encryption is an essential and necessary factor when storing data on the cloud where only the owner of the data can access the data, so the correct choice of the encryption algorithm is necessary for the cloud providers and users also where more efficiency and security accuracy is available [10].

\section{STATEMENT OF THE PROBLEM}

Cloud providers provide many services, including applications and storage many companies and users do not trust the providers of these services due to security concerns. Where the user does not upload his personal data to the cloud because the cloud providers are able to read and modify every bit loaded on the cloud and use it for personal purposes, and this thing does not comply with respecting the user's privacy. Furthermore, some cloud providers still use traditional security techniques that are not secure with low-security level to protect user privacy. Some of the cloud providers have started to use high-level technologies to protect the privacy of users and the security of their data, but there remains a problem that the provider of the cloud itself is still able to access user data, and this is not safe for users. This problem can be solved when following FHE systems when storing data on the cloud where these systems can encrypt 
the data and store it in the cloud in an encrypted form and thus the cloud provider or others cannot see the data and use it, so the privacy of users and the security of their data are protected.

\section{RELATED WORK}

A symmetric encryption system was introduced to provide more data security and protect it from any serious attack in the year 2019 by [11], And about two years before that, specifically in (2017 )data security problems were presented when stored in the cloud and a method was proposed to provide complete data security using AES encryption technology with the use of standard encryption 128 Bit by [12],in (2018) an encrypting system was introduced based on the Pailler algorithm that supports the addition process and on the RSA algorithm that supports the multiplication process on the encrypted data by [13], An encrypting scheme based on a pattern called asymmetric cipher padding (OAEP) was introduced with the symmetric cipher algorithm that stands for the RSA algorithm in (2018) by [14], and a completely symmetric encrypting system based on Euler's theory has been introduced and time complexity has been calculated and compared to other methods the size of an encryption key up to bits in (2018) by [15], while the size of the encryption key in our algorithm reaches more than 2048 bits and the encryption process is accomplished through more complicated and powerful mathematical equations, in (2018) a completely symmetric encrypting system was introduced on that relies the principle of changing a number from the plain text to another number using a secret key without converting On binary format then compare the result with DGHV and SDS systems by [16]. Not all banks use online banking services despite the tremendous benefits they enjoy due to the attacks they are subject to by cybercriminals. [17], [18] The authors present many attacks that occur on different components of online banking services, such as Spy_Eye Malware. Fraud and educational phishing are among the most common attacks on banking services, as these attacks steal user login confidentiality. [19]-[23] Researchers offer many possible solutions to phishing and attacks within browsers and across sites, but without these solutions fix the cloud-based environments. Also, risks related to banking services jobs were presented by researchers in [24].

\section{BANK SERVICES IN THE CLOUD}

Because of the limited use of cloud services by companies in various fields and banks, it has also created a strong incentive for cloud services providers to develop their services, especially security, as researchers in cloud affairs have been stimulated to intensify their research and efforts to find appropriate solutions for bank safety and information. The use of cloud services for banks is considered a dangerous matter to some extent because to this day storing data on the cloud is not considered a safe matter because when the data is uploaded to the cloud, control over customer data (such as account numbers, deposits, etc.) is lost, but on the other hand, there are many reasons makes banks and other institutions to use cloud services, whether public or private, including scalability, agility and saving many costs, but these benefits come with risks related to data security, you should consider these risks when using cloud services. This problem can be solved if cloud providers use strong encryption algorithms when storing customer data in the cloud. This risk is illustrated by US national law [25]. Most cloud service providers who use encryption algorithms require their customers to trust them and use their decryption keys when making any modification to their previously stored data. This does not fit with the principle of respecting the privacy of customer data security. Given the high costs of computers, the recent financial crisis, and current health conditions (COVID-19), Banks must reduce their information technology costs, but this should not be done at the expense of data security and integrity. All these reasons drive banks to use cloud services. This paper offers a simplified banking system for storing data on the cloud, this system relies on A New Asymmetric Fully Homomorphic Encryption Scheme, as this algorithm relies on data encryption, storage on the cloud and modification on request without the need to decrypt data and own a private secret key for customers, and thus the privacy of customer data security has been respected and therefore customer data (banks) on the cloud is encrypted and cannot be 
viewed Anyone who is not authorized is required. Our new algorithm is explained further in the remainder of this paper.

\section{HOMOMORPHIC ENCRYPTION CATEGORIES}

There are three main categories of Homomorphic encryption schemes: Partially Homomorphic Encryption PHE, Somewhat Homomorphic Encryption SWHE, and Fully Homomorphic Encryption FHE schemes. PHE schemes, such as RSA [8], ElGamal [26], Paillier [9], Etc., allow to applying either addition or multiplication on encrypted data. G. Kalpana et al. [27], allowing unlimited additions and a single multiplication. Construction of scheme supporting both operations addition and multiplication simultaneously is possible in 2009 by Gentry [28] by using fully homomorphic encryption.

\subsection{Partially Homomorphic Encryption (PHE)}

An encryption technique is called a Partially Homomorphic Encryption (PHE) if it applies only one operation on encrypted data, i.e., either addition or multiplication but not both [29].

\subsection{Somewhat Homomorphic Encryption (SWHE)}

The scheme that supports a limited number of homomorphic operations known as somewhat homomorphic encryption [30]. An encryption technique is called Somewhat Homomorphic encryption (SWHE) if it performs a limited number of addition and multiplication operations on encrypted data.

\subsection{Fully Homomorphic Encryption (FHE)}

An encryption technique is called Fully Homomorphic (FHE) if it performs both addition and multiplication simultaneously and can compute any operation [6].

\section{PROPERTIES OF HOMOMORPHIC ENCRYPTION}

\subsection{Additive Homomorphic Encryption:}

A homomorphic encryption is additive if:

$E n c(m 1 \oplus m 2)=\operatorname{Enc}(m 1) \oplus E n c(m 2) .(1)$

\subsection{Multiplicative Homomorphic Encryption:}

A homomorphic encryption is multiplicative, if:

$E n c(m 1 \otimes m 2)=E n c(m 1) \otimes E n c(m 2) .(2)$

\section{FERMAT AND EULER THEOREMS}

Two important theorems presented the first by Pierre de Fermat and the second by Leonhard Euler. Both theorems are related to powers in modular arithmetic.

Fermat's Little Theorem

Suppose that $\mathrm{p}$ is prime and $\operatorname{gcd}(\mathrm{a}, \mathrm{p})=1$ (or a and $\mathrm{p}$ are relatively prime or $\mathrm{p}$ does not divide, then

$\mathrm{M}^{\mathrm{p}-1} \equiv 1(\bmod \mathrm{p})$

\subsection{Euler's Theorem}

Euler's Theorem is a generalize of Fermat's Little Theorem. Suppose $n$ be an arbitrary positive integer, $\varnothing(\mathrm{n})$ denote the number of integers $1=<\mathrm{a}<=\mathrm{n}$ such that if $\operatorname{gcd}(\mathrm{a}, \mathrm{n})=1$,

then: $\mathrm{M}^{\phi(\mathrm{n})} \equiv 1(\bmod \mathrm{n}) \quad(4)$

So that:

$\mathrm{M}^{\mathrm{r}^{*} \emptyset(\mathrm{n})+1} \equiv \mathrm{M}(\bmod \mathrm{n})$, when $\mathrm{r}$ is an integer, $\mathrm{M}<\mathrm{n}$ and $\mathrm{n}=\mathrm{p}^{*} \mathrm{q}$ where $\mathrm{p}$ and $\mathrm{q}$ are two primes number. 


\section{PROPOSED FULLY HOMOMORPHIC ENCRYPTION SYSTEM}

8.1 The proposed scheme works as follows:

Generating the encryption key and then encrypting the numbers and texts and storing them in encrypted form on the cloud. In our work, we use a local cloud and experiment with the proposed scheme on it. The purpose of this process is to save the data encrypted on the cloud so that no one can view the data and use it for personal purposes, Therefore, when the data owner needs to perform an amendment of the encrypted data on the cloud, an encrypted request is sent to the server and the server performs mathematical operations on the encrypted data and returns an encrypted result where this encrypted result can only be decrypted through the private encryption key which is with the owner of Data only so that he can decrypt the encrypted result and see his data. In this way, we have maintained the privacy and security of the data when stored in the cloud. These procedures go through three stages. Generation the encryption key stage, the encryption stage, and the decryption stage.

\subsection{Algorithm of A New Asymmetric Fully Homomorphic Encryption \\ - Key Generation:}

Generate two large Prime number $\mathrm{p}, \mathrm{q}$

Select two big random integer $\mathrm{z}$ and $\mathrm{w}$

Compute $n=p * q$ and $\varnothing(n)=(p-1) *(q-1)$

Calculate $=n * Z, e k=w * \varnothing(n)$ and Pubkey $=P k * e k$

The public key is $\left(\mathrm{Pub}_{\mathrm{key}}, \mathrm{ek}\right)$ and private key is $(\mathrm{p}, \mathrm{q})$

- Messages Encryption:

The message $M$ will always be less than $\mathrm{p}_{\mathrm{k}}$, that $\left(\mathrm{m}_{1} \& \mathrm{~m}_{2}\right),\left(\mathrm{m}_{1}+\mathrm{m}_{2}\right)$ and $\left(\mathrm{m}_{1} * \mathrm{~m}_{2}\right)<$ $\mathrm{P}_{\mathrm{k}}$

The schema of message encryption is:

$\boldsymbol{C}=\boldsymbol{P u} \boldsymbol{b}_{k e y}+\boldsymbol{m}^{\boldsymbol{e}_{k+1}} \boldsymbol{\operatorname { m o d }} \boldsymbol{P}_{\boldsymbol{k}}$

Which depended only public-key (Pub $\left.\mathrm{P}_{\mathrm{key}}, \mathrm{ek}, \mathrm{Pk}\right)$ to send the message

Where

M: Plain-text(Message), C: cipher-text

- Message Decryption:

The schema of cipher decryption is:

$M=C \bmod n$

\subsection{Evaluate of fully Homomorphic Encryption Properties}

\subsubsection{To Proof correctness of the scheme:}

$\mathrm{C}=\mathrm{Pub}_{\mathrm{key}}+\mathrm{M}^{\mathrm{ek}+1} \bmod \mathrm{P}_{\mathrm{k}}$

$\mathrm{M}=\mathrm{C} \bmod \mathrm{n}=\mathrm{Pub}_{\mathrm{key}}+\mathrm{M}^{\mathrm{ek+1}} \bmod \mathrm{P}_{\mathrm{k}}(\bmod \mathrm{n})$

$=P_{\text {ubey }}(\bmod n)+M^{\mathrm{ek+1}} \bmod n\left(\bmod P_{k}\right)$

$=0+\mathrm{M}^{\mathrm{e}+1} \bmod \mathrm{n}\left(\bmod \mathrm{P}_{\mathrm{k}}\right)=\mathrm{M} \bmod \mathrm{P}_{\mathrm{k}}=\mathrm{M}\left(\right.$ since $\left.\mathrm{M}<\mathrm{P}_{\mathrm{k}}\right)$

\subsubsection{The Proof of Additive Homomorphic}

If the following condition is fulfilled, it becomes clear to us that the proposed scheme Additive Homomorphic:

$m_{1}+m_{2}=\operatorname{dec}\left[\operatorname{enc}\left(m_{1}\right)+\operatorname{enc}\left(m_{2}\right)\right]$

Where dec is the decryption function and enc is the encryption function

Proof:

$$
\begin{aligned}
& c_{1}=P u b_{k e y}+m_{1}{ }^{e_{k+1}}\left(\bmod P_{k}\right)=P_{k} * e_{k}+m_{1}{ }^{e_{k+1}}\left(\bmod P_{k}\right) \\
& c_{2}=P u b_{k e y}+m_{2}{ }^{e_{k+1}}\left(\bmod P_{k}\right)=P_{k} * e_{k}+M_{2}{ }^{e_{k+1}}\left(\bmod P_{k}\right) \\
& c_{1}+c_{2}=\left[P_{k} * e_{k}+m_{1} e_{k+1}\left(\bmod P_{k}\right)+P_{k} * e_{k}+m_{2} e_{k+1}\left(\bmod P_{k}\right)\right] \bmod \mathrm{n} \\
& =P_{k} * e_{k}(\bmod n)+m_{1} e_{k+1}(\bmod n) \bmod P_{k}+P_{k} * e_{k}(\bmod n)+ \\
& m_{2} e_{k+1}(\bmod n) \bmod P_{k}=\mathrm{m}_{1}+\mathrm{m}_{2} \\
& {\left[m_{1}{ }^{e_{k+1}}(\bmod n) \bmod P_{k}=\mathrm{m}_{1} \bmod P_{k}=\left[m_{1}\right],\right. \text { by Fermat's Little Theorem }}
\end{aligned}
$$


$\left(\mathrm{m}_{1}{ }^{\mathrm{r}^{*} \boldsymbol{\theta}(\mathrm{n})+1} \bmod \mathrm{n} \equiv \mathrm{m}_{1}\right)$

$\left[P_{k} * e_{k}(\bmod n)=0\right]$, since $P_{k}=\mathrm{n} * \mathrm{z}$, which is multiple of $\mathrm{n}$ modular $\mathrm{n}$ equal to zero.

And so on the same for other terms.

\subsubsection{The Proof of Multiplicative Homomorphic}

If the following condition is fulfilled, it becomes clear to us that the proposed scheme Multiplicative Homomorphic:

$\mathrm{m}_{1} * \mathrm{~m}_{2}=\operatorname{dec}\left[\mathrm{enc}\left(\mathrm{m}_{1}\right) *\right.$ enc $\left.\left(\mathrm{m}_{2}\right)\right]$

Where dec is the decryption function and enc is the encryption function

Proof:

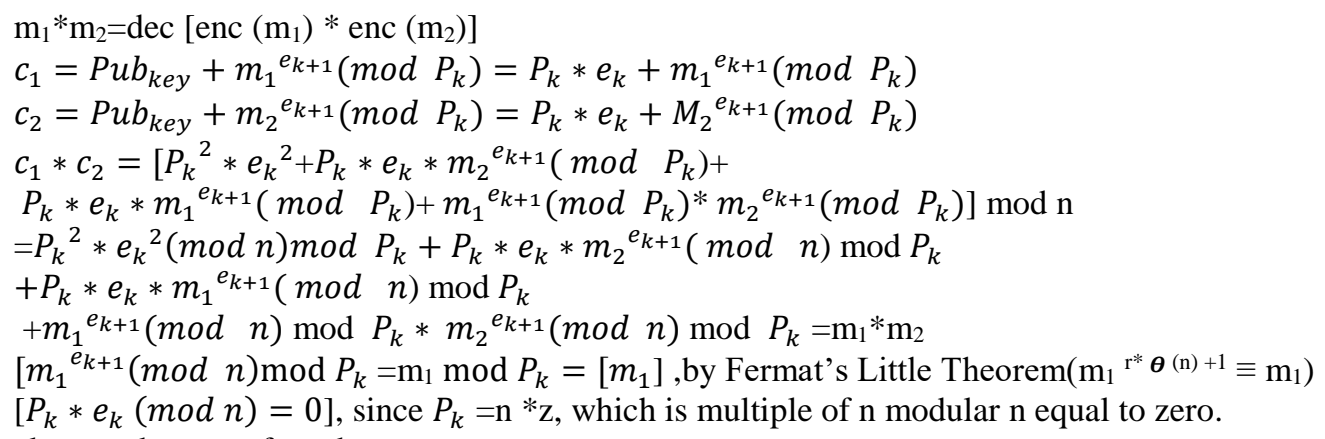

And so on the same for other terms.

\section{RESULTS AND DISCUSSION}

Our proposed method has been applied in Java Language on a laptop that has these characteristics Intel (R) core (TM) i7-8550U CPU @1.80GHz 2.00GHz, 8 GB Ram, 64-bit Operating System, x64-based processor, Windows 10 and Big Integer library of java is used.

\subsection{Case studies}

In this section, several studies will be presented that we conducted to test our system to prove the creation of the secret key and its use for encryption and decryption

\section{Case study 1:}

Let us choose two different number $\mathrm{m}_{1}=4, \mathrm{~m} 2=8$, select prime numbers $\mathrm{p}=467, \mathrm{q}=307$, select random number $\mathrm{r}=401, \mathrm{z}=271$ and $\mathrm{w}=449$ and compute $\mathrm{n}, \varnothing(\mathrm{n})=(\mathrm{p}-1) *(\mathrm{q}-1)$, pk,ek and Pub key where $\mathrm{n}=\mathrm{p}^{*} \mathrm{q}, \mathrm{pk}=\mathrm{n}^{*} \mathrm{z}, \mathrm{ek}=\mathrm{w}^{*} \mu(\mathrm{n})$ and $\mathrm{Pub}_{\mathrm{key}}=\mathrm{pk} * \mathrm{ek}$, as in figure 1 , so $\mathrm{n}=57490969$,

$\varnothing(\mathrm{n})=57038400, \mathrm{pk}=15580052599, \mathrm{ek}=25610241600$ and $\mathrm{Pub}_{\text {key }}=399008911201097918400$

now we will compute $\mathrm{c}_{1}, \mathrm{c}_{2}$ where

$$
\begin{aligned}
& \mathrm{c}_{1}=\text { Pub }_{\mathrm{key}}+\mathrm{m}_{1}{ }^{\mathrm{ek}+1} \bmod \mathrm{pk} \\
& \mathrm{c}_{1}=399008911201097918400+4^{25610241600+1} \bmod 15580052599 \\
& \mathrm{c}_{1}=399008911209721563754 \\
& \mathrm{c}_{2}=\text { Pub }_{\mathrm{key}}+\mathrm{m}_{2}{ }^{\mathrm{ek}+1} \bmod \mathrm{pk} \\
& \mathrm{c}_{2}=399008911201097918400+8^{25610241600+1} \bmod 15580052599 \\
& \mathrm{c}_{2}=399008911201097918408
\end{aligned}
$$

\section{A. Check the Additive Homomorphism}

Let us define $\mathrm{C}_{3}$ is the result of $\mathrm{c}_{1}+\mathrm{c}_{2}$

$$
\begin{aligned}
& \mathrm{c}_{3}=\mathrm{c}_{1}+\mathrm{c}_{2} \\
& \mathrm{c}_{3}=399008911209721563754+399008911201097918408 \\
& \mathrm{c}_{3}=798017822410819482162 \\
& \mathrm{~m}_{3}=\mathrm{c}_{3} \bmod \mathrm{p} \\
& \mathrm{m}_{3}=798017822410819482162 \bmod 467 \\
& \mathrm{~m}_{3}=12, \text { which is the same of } \mathrm{m}_{1}+\mathrm{m}_{2}=4+8=12
\end{aligned}
$$




\section{B. Check the Multiplication Homomorphism}

Let us define $\mathrm{C}_{4}$ is the result of $\mathrm{C}_{1} * \mathrm{C}_{2}$

$\mathrm{c}_{4}=\mathrm{c}_{1} * \mathrm{c}_{2}$

$\mathrm{c}_{4}=399008911209721563754 * 399008911201097918408$

$\mathrm{c}_{4}=159208111221326555237218115498200062183632$

$\mathrm{m}_{4}=\mathrm{C}_{4} \bmod \mathrm{p}$

$\mathrm{m}_{4}=159208111221326555237218115498200062183632 \bmod 467$

$\mathrm{m}_{4}=32$, which is the same of $\mathrm{m}_{1} * \mathrm{~m}_{2}=4 * 8=32$

\section{Case study 2:}

We took as an example of 2048 bit A message containing several languages: English, Kurdish, Arabic and Chinese, to indicate that our scheme works in all languages. The message was:

Fully Homomorphic encryption system is the best solution when storing data in the cloud

التشفير التمانلي التام يعد افضل حل عند تخزين البيانات على السحابة

全同态加密系统是在云中存储数据时的最佳解决方案

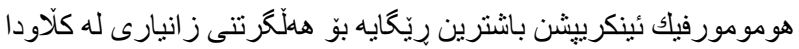

p=17677407741877305812974453795695829556481143458816523982107269504893610803

58551018895584377087249191335724085433447024543185504758455631650550492203454 64241056074079024109166204002367569192042605346490577631743669656928602593119 87269878821499594667240449799539516699641411229698173419572164662026446722542 45842179370658749263407810196728233031159967134374914299433764943853524262950 10248374434570101830938140507177653834149747566763219105733110623512036996321 94535963913255933743321718394914382531286601992742907837501385111059060019874 77588021055291849648949570048628664064077590484738933338889181212587636009178 3321

q=27479324216009061896292077972720073521672478547125991911969838200015924859 13445493592485385233195477049349741424361315108262249184165808418072920022405 32191240404858029386958419842799686682471795353968158406182041332189135779311 42268145416491773923591539609233733196404783888133066336919076387688113196684 53317377733188135302564932982125876956345691771505946023899771659248597752163 70450129344735564036443811888747396621308549176665800328009187819795087471726 72324090656507275368384213216100552615957975944972170017248687813245723168819 51160642892464178663003766675944121364487852226241867052819190294856408013991 2399

$\mathbf{r}=221704313573415863159846363027780946932749477453300762390358010519665644221$ 87140855682320560549672999017524704999360401622672733792707046542939076114622 71294645777433048961662607368834573356607007276968285901809569193575593361195 95183592425870109416627348226330225417095167338356961355353440978586915860732 25295416801346356644268401349085223233791565552540400714669486000596164658328 53354843569032953795841177317760938909217583755697157793455522895018625285194 62537280823951426545206331004884970981539080382288397414773455434023413361954 47440852503074070578011632695922887511174829871285336692705513320415788577077 679

$\mathbf{z}=275249857110002837815800172710466356018329806961099866732193189637794181023$ 63701442691287193897733121259027100064136824329301210609187447135330014261476 50949829649323789524585055409208200878901235351905015521587034028332528553850 11614529987564845152327785052325832471682154173508783558623331252252968152219 16027089844912780553832369867433821093674360804418144757102515585490143240810 04911834111030009876069187484163346362681914347253110719669693609577828580519 55506648516062732533087705315351585063502912220372815271233309365462638783981 32239648469349585337867535450178014597005244053675435612025992973391019104165 051

$\mathbf{w}=27970928086245618554130758033009128606102344747844162488872725298013994846$ 66750703759209951503644756559344326593436488713439727941370882474185729394202 
26568930125038906366151005406608696506059925021656511039066192720032347527969 16825734793075507607756350581033260197597066561939143858829691043997062844545 31619047315794357733653339906082961824373494521550887529988893790465408818017 14334723034181238734693852986203812112960241219323273443674522837689161421055 48731309980467698859425343574740941094513723675441376882831853221735200060302 65054894069344437565316320341105324384528818788195486201847329573292754450278 3547

$\mathbf{n}=10769580094727002503471943187166493982427611244942262080176583144009934659$ 81143042618421771243562508741529558462567496164974218162794828070090102743116 14675744981617706981159659278840348642493533661619004418505278308630146273559 49776532784746004292342193106772858086635741189742878628382470222788825482078 61943946691808699382242542777997373518125113923399713103380525808288086413349 19212781469514351491835805699434775073115335471855882883646035920190250024882 589632915694420312521092543951867067207925855181429097843652411555488444983342 83189320986270780236136127138605362150211782152865745381504375705543553816512 21080065655104503709942786890696056308249476633106547878851299305806005512585 835413932764632370967194097903004685319180131254616994241643641137334990007492 38417041896555095544181828755557265971346981037514011439434062675852888528243 74771452124934839087438528317526026501724778542161519207350378280716665713992 33093893068896685065293401056907219377231430933622052700439764141956013176800 08441865070124950005050281952689339441458657533442962099842432475896256930594 04126544402701922750498619154017022505738530444115173928551210212914269478804 12860202143331118919677011230626375006094163641104605963225042768615177326418 04068211108887647811534025697434933102079051297094993378106248264111141798110 06075632477579241815254478917115784613782075604252933691662961011635444393974 09530862792837844730182746315230788913885544817429507257108778271967617395803 69155228561187843144824186591120699659377823199894425771268810353616296436945 83356611341470626782237814429171429730166442379827572105352396392849523835926 02752329293726120327361136868710322352784471970286028331332376113647464712430 52579019338540424638344553771108463500791094165293405274249922836843857047119 94952998965052165507568745669931336591389786290832307146403761468886675257507 99641

\section{The Message after encryption (Cipher Text):}

89295900219191118519022931543915293570526367958119369756690615872509965393822 31001740321331646477256733520778478120002484749463886513532178306089038239543 02840563285267128770795997132212844773626461847056101966474470314533455350620 35961583853467335353692058643877483368839024324323253148702413976549021747125 84267662290418187689873676027208635786466580431611882199972233892265537394995 11808792503513297027206315025447067373256990258810928316926704967052369318515 39123933129291735790734203167224718452036002934602397499396928658480040019814 48753794055536037412565397913390978373800211854455912862135884453189535416422 48643350177392070083256386839997606638372180955482018837827049995130280291737 80477854749578970665250919555137362702387050753022510647671078135904779199076 99613784625827003099595182929464504486913045408507958278445397409776501583072 08218365845189684157830888068028334654562249806532825451487522249699122205996 68854356943638262766852210468449630191908418239367807625716544138399309127329 81414556161366337124764581505287880141762366994590146327071125276854185469427 99348536482500412527825762989106752138717310011092660740968429827301331409068 425432594068827865752883705999301179366129459535713247981398667656227466682555 71132508113770654369759346831687920038271029897667892423374810263220958410482 11999897930106631362581295255946251804804094917660182672337363831278103290372 31174843643462187324258096511620171705278849528849072515299421288789465771681 
43248593387968474598670964355373653052749795427143289149329468840372409439550 54849884901788502012854967911585319237252603534567463085636877395614784177845 96965108486808004477401942768168047222903019793219239964275477699956509410684 52328268610784830191025845961252703855535813829958762877983640392413802255355 46428135007324080259252467858536957855977571160173001816724564278149168611602 45823522934535900592968120713467599445675667134123013501889780848602863778123 26326135402160036272556155249730667204639551120185330579724202900318999797435 42879664943985365924734927922050496995004574143976224500409164715995045548258 80591841522178517562068523430265973738052677570728032144342485814823604886324 50637171202401642868793240782799510215265259095894494535471354049093275530862 39224260691868986164678833953163322689297576670114211557653732496694098293514 17825457316748989715570095449667266994555193687741260632502600000910540602742 76914747724897228602651401673101955313890555932552792140290794613348372108415 57663927723446685822998162225135864218539770247362377169461300200659677780338 82526148523095531246278362277516264723046543270412030208182692229566558800145 51506581473160603366883980285215638955963339387612200134501841503508805535949 21185510472589502996007692047569736831254613398327847474835174479696247965785 $6968471415060827235319400271780 \ldots$...etc Due to the length of the encrypted text (Cipher Text), which reaches more than 530 pages, it has been truncated. Where the encrypted time was $151225 \mathrm{~ms}$ and the decrypted time was $157 \mathrm{~ms}$. we have also tested it on text with 8KB in its size and several different Keys in terms of size, and we compared the results with the planners from in terms of velocity, we obtained the following results as shown in table 1 and figure 1

Table 1: Performance of small file size encryption and decryption measured in a millisecond with 64bit key length.

\begin{tabular}{ccc}
\hline File size & Encryption time $(\mathbf{m s})$ & Decryption time $(\mathbf{m s})$ \\
\hline 8 kilobyte & $444 \mathrm{~ms}$ & $\mathbf{5 4} \mathbf{~} \mathbf{~}$ \\
\hline 12 kilobyte & $517 \mathrm{~ms}$ & $\mathbf{5 6} \mathbf{~ m s}$ \\
\hline 20 kilobyte & $1262 \mathrm{~ms}$ & $\mathbf{2 0 9} \mathbf{~ m s}$ \\
\hline 28 kilobyte & $1705 \mathrm{~ms}$ & $\mathbf{3 8 5} \mathbf{~ m s}$ \\
\hline 40 kilobyte & $2504 \mathrm{~ms}$ & $\mathbf{8 1 8} \mathbf{~ m s}$ \\
\hline
\end{tabular}

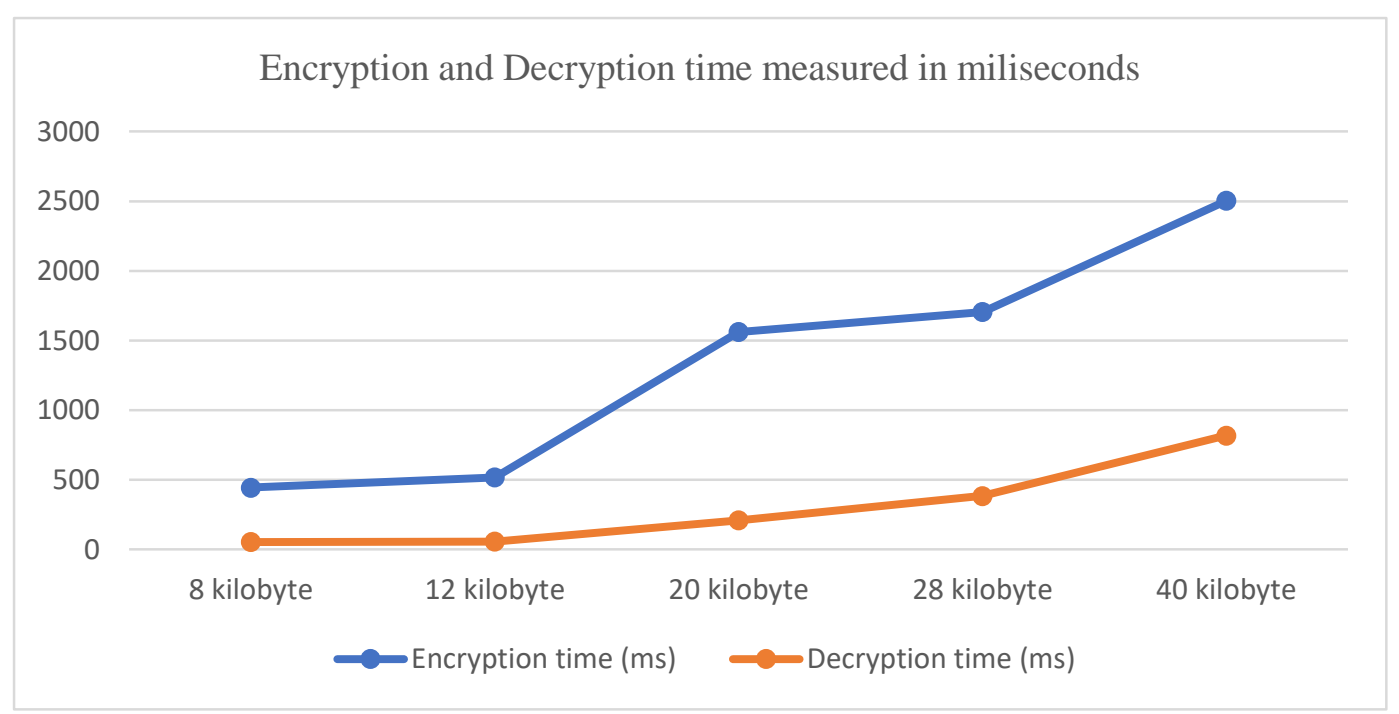

Figure 1: Computation encryption and decryption time of our schema 


\subsection{Our Asymmetric Fully Homomorphic Encryption Scheme Applied to Banking Data in}

the cloud:

The Bank Application work as shown in figure 2

9.3 Cloud and banking app experiences:

As for our banking application, we created two accounts and encrypted them with a 2048-bit encryption key using our previously mentioned algorithm which required 1264 milliseconds as encryption time and stored it on a local private cloud as shown in figures 3 and 4 . And also requests 65 milliseconds as the decryption time as is shown in figures 5 and 6

\subsection{Results of NIST Statistical Tests on the Generated Secret Keys:}

The randomness of this novel proposal is evaluated by the well-known NIST test suite[31]. Table 2 shows the test results of the proposed algorithm from the NIST statistical tests, demonstrating that the best statistical performance was obtained with this algorithm.

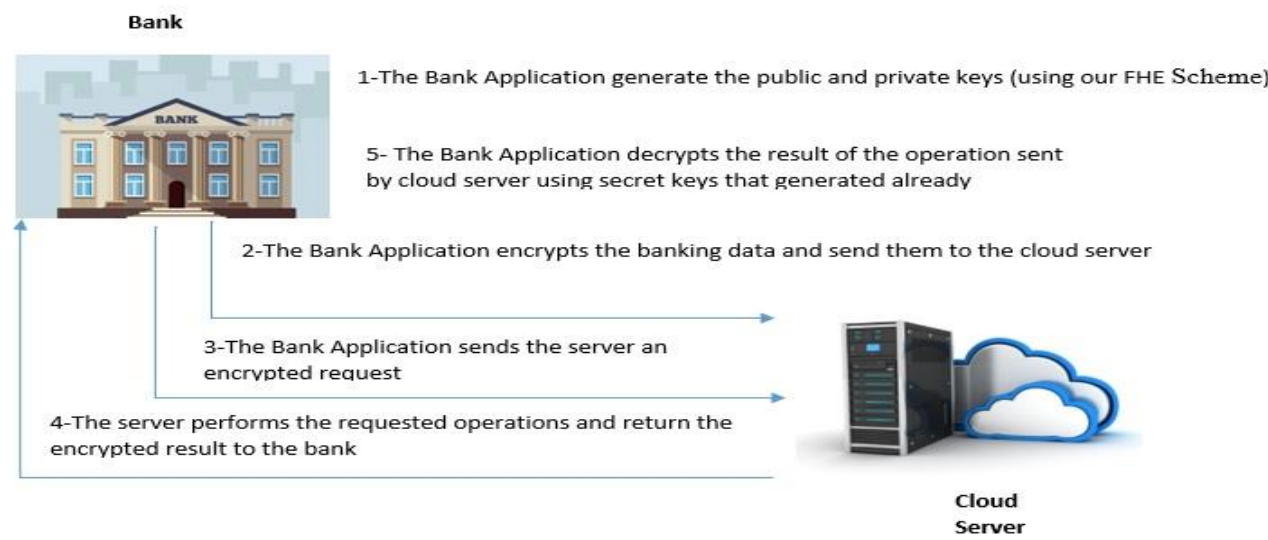

Figure 2: Scheme Representing the Link between the Bank and its Data Hosted in a Cloud Provider Server

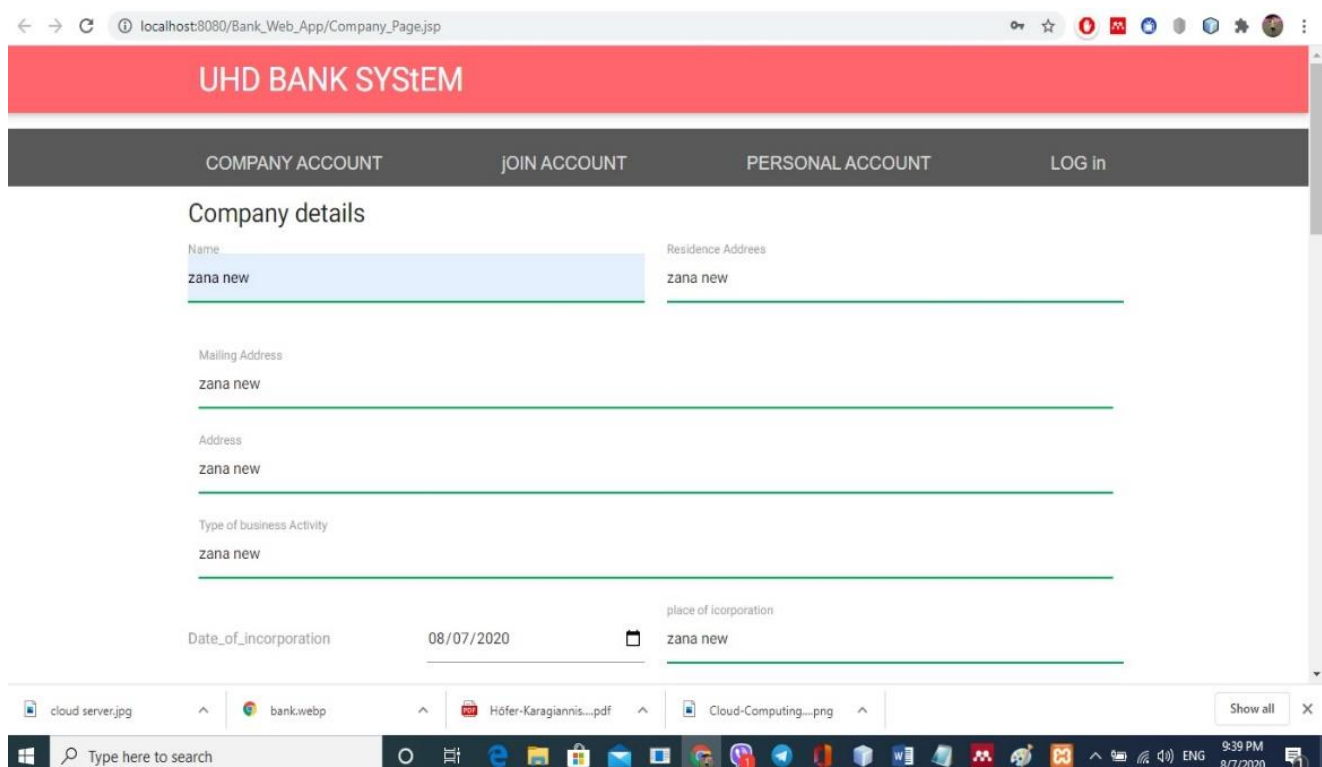

Figure 3: Create a new account and encrypt it at Bank Application Level then send to the cloud server 


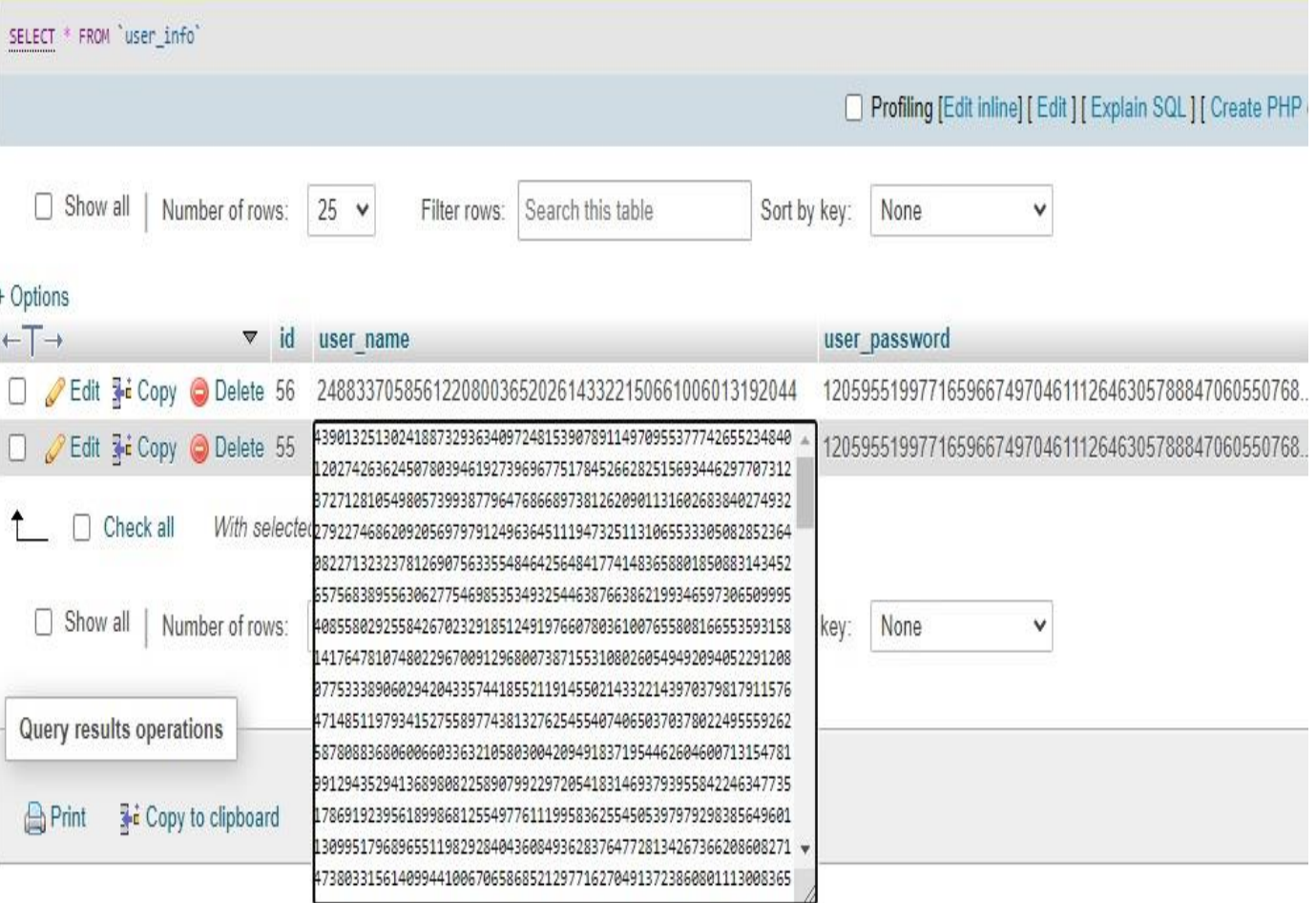

Figure 4: User login data in encrypted form at Cloud Server leve

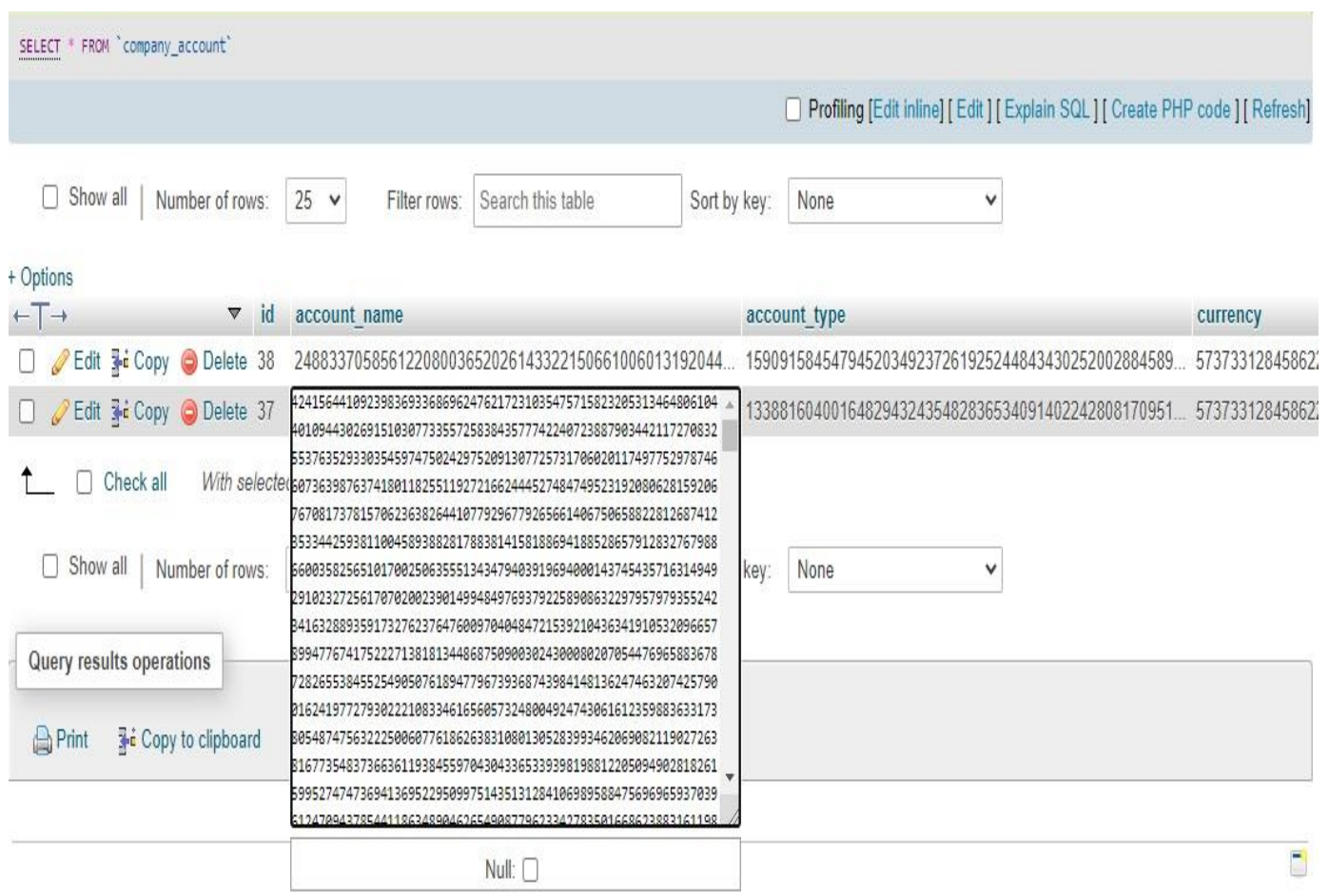

Figure 5: User Data in encrypted form at Cloud Server 
Figure 6: User Data at Bank Application Level after decrypting it

Table 2: NIST test results for the proposed algorithm

\begin{tabular}{|c|c|c|}
\hline Tests & P-value & Result \\
\hline Frequency (Monobits) & 0.969730 & SUCCESS \\
\hline Block Frequency & 0.934397 & SUCCESS \\
\hline Cumulative Sums (Cusum) & 0.955178 & SUCCESS \\
\hline Runs & 0.791649 & SUCCESS \\
\hline Longest Run of Ones & 0.812369 & SUCCESS \\
\hline Rank & 0.828802 & SUCCESS \\
\hline Discrete Fourier Transform & 0.749568 & SUCCESS \\
\hline Non-Overlapping Template Matching & 0.865923 & SUCCESS \\
\hline Overlapping Template Matching & 0.667917 & SUCCESS \\
\hline Approximate Entropy & 0.879027 & SUCCESS \\
\hline Random Excursions & 0.919402 & SUCCESS \\
\hline Random Excursions Variant & 0.942381 & SUCCESS \\
\hline Serial & 0.842202 & SUCCESS \\
\hline Linear Complexity & 0.909160 & SUCCESS \\
\hline
\end{tabular}




\section{CONCLUSION}

In this paper, a new encryption technology based on Asymmetric(public key) Fully Homomorphic Encryption Scheme has been proposed to ensure the security of user data when stored on the cloud and respect their privacy at rest that support all languages like(English, Arabic, Kurdi and Chinese) and others, Very large prime numbers (up to 617 digits, 2048 bit) represent the strength for attack of our scheme because the proposed system depends on the problem of Factorization to the primary factors, which are considered mathematical issues under discussion at the present time and the user data is encrypted with different keys and thus provides effective security which prevents attackers from analyzing it and using it for personal purposes that ensure the security measures of the proposed technology it is resistance For any kind of brute force, mathematics, and time attacks, it explains that it can protect user data even if it is leaked to unauthorized parties, thus the proposed scheme ensures data security when it is stored in the cloud.

\section{REFERENCE}

[1] B. Varghese and R. Buyya, "Next generation cloud computing: New trends and research directions," Futur. Gener. Comput. Syst., vol. 79, pp. 849-861, 2018, doi: 10.1016/j.future.2017.09.020.

[2] N. Subramanian and A. Jeyaraj, "Recent security challenges in cloud computing," Comput. Electr. Eng., vol. 71, no. June, pp. 28-42, 2018, doi: 10.1016/j.compeleceng.2018.06.006.

[3] M. Masud and M. Shamim Hossain, "Secure data-exchange protocol in a cloud-based collaborative health care environment," Multimed. Tools Appl., vol. 77, no. 9, pp. 11121-11135, 2018, doi: 10.1007/s11042-0175294-5.

[4] P. R. Kumar, P. H. Raj, and P. Jelciana, "Exploring Data Security Issues and Solutions in Cloud Computing," Procedia Comput. Sci., vol. 125, no. 2009, pp. 691-697, 2018, doi: 10.1016/j.procs.2017.12.089.

[5] A. Suresh and R. Varatharajan, "Competent resource provisioning and distribution techniques for cloud computing environment," Cluster Comput., vol. 22, pp. 11039-11046, 2019, doi: 10.1007/s10586-017-12936.

[6] M. A. Mohammed and F. S. Abed, "A symmetric-based framework for securing cloud data at rest," Turkish J. Electr. Eng. Comput. Sci., pp. 347-361, 2019, doi: 10.3906/elk-1902-114.

[7] A. Acar, H. Aksu, A. S. Uluagac, and M. Conti, "A Survey on Homomorphic Encryption Schemes," ACM Comput. Surv., vol. 51, no. 4, pp. 1-35, 2018, doi: 10.1145/3214303.

[8] Rohini and T. Sharma, "Proposed hybrid RSA algorithm for cloud computing," Proc. 2nd Int. Conf. Inven. Syst. Control. ICISC 2018, no. Icisc, pp. 60-64, 2018, doi: 10.1109/ICISC.2018.8398902.

[9] M. E. Zhao and Y. Geng, "Homomorphic Encryption Technology for Cloud Computing," Procedia Comput. Sci., vol. 154, pp. 73-83, 2018, doi: 10.1016/j.procs.2019.06.012.

[10] C. Stergiou, K. E. Psannis, B. B. Gupta, and Y. Ishibashi, "Security, privacy \& efficiency of sustainable Cloud Computing for Big Data \& IoT," Sustain. Comput. Informatics Syst., vol. 19, pp. 174-184, 2018, doi: 10.1016/j.suscom.2018.06.003.

[11] S. Kaushik and A. Patel, "Scheme," 2019 4th Int. Conf. Internet Things Smart Innov. Usages, pp. 1-6, 2019.

[12] M. P. Babitha and K. R. R. Babu, "Secure cloud storage using AES encryption," Int. Conf. Autom. Control Dyn. Optim. Tech. ICACDOT 2016, pp. 859-864, 2017, doi: 10.1109/ICACDOT.2016.7877709.

[13] X. Song and Y. Wang, "Homomorphic cloud computing scheme based on hybrid homomorphic encryption," 2017 3rd IEEE Int. Conf. Comput. Commun. ICCC 2017, vol. 2018-Janua, pp. 2450-2453, 2018, doi: 10.1109/CompComm.2017.8322975.

[14] D. Das, "Secure cloud computing algorithm using homomorphic encryption and multi-party computation," Int. Conf. Inf. Netw., vol. 2018-Janua, pp. 391-396, 2018, doi: 10.1109/ICOIN.2018.8343147.

[15] S. S. Hamad and A. M. Sagheer, "Fully Homomorphic Encryption based on Euler's Theorem," J. Inf. Secur. Res., vol. 9, no. 3, p. 83, 2018, doi: 10.6025/jisr/2018/9/3/83-95.

[16] S. S. Hamad and A. M. Sagheer, "Design of fully homomorphic encryption by prime modular operation," Telfor J., vol. 10, no. 2, pp. 118-122, 2018, doi: 10.5937/telfor1802118H.

[17] K. J. Hole, V. Moen, and T. Tjostheim, "Case study: Online banking security," IEEE Secur. Priv., vol. 4, no. 2, pp. 14-20, 2006, doi: 10.1109/MSP.2006.36.

[18] C. Ronchi, A. Khodjanov, M. Mahkamov, and S. Zakhidov, "Security, privacy and efficiency of internet banking transactions," World Congr. Internet Secur. WorldCIS-2011, pp. 216-222, 2011, doi: 10.1109/worldcis17046.2011.5749854.

[19] M. Ystenes, "ET gone phishing," New Sci., vol. 217, no. 2903, p. 33, 2013, doi: 10.1016/S02624079(13)60368-1.

[20] J. Zhan and L. Thomas, "Phishing detection using stochastic learning-based weak estimators," IEEE SSCI 2011 Symp. Ser. Comput. Intell. - CICS 20112011 IEEE Symp. Comput. Intell. Cyber Secur., pp. 55-59, 2011, doi: 10.1109/CICYBS.2011.5949409.

[21] S. Ranjan and E. Knightly, "High performance distributed Denial-of-Service resilient web cluster 
architecture," NOMS 2008 - IEEE/IFIP Netw. Oper. Manag. Symp. Pervasive Manag. Ubiquitous Networks Serv., pp. 1019-1024, 2008, doi: 10.1109/NOMS.2008.4575272.

[22] F. Bin Mat Nor, K. Abd Jalil, and J. L. Ab Manan, "An enhanced remote authentication scheme to mitigate man-in-the-browser attacks," Proc. 2012 Int. Conf. Cyber Secur. Cyber Warf. Digit. Forensic, CyberSec 2012, pp. 271-276, 2012, doi: 10.1109/CyberSec.2012.6246086.

[23] F. Kerschbaum, "Simple cross-site attack prevention," Proc. 3rd Int. Conf. Secur. Priv. Commun. Networks, Secur., pp. 464-472, 2007, doi: 10.1109/SECCOM.2007.4550368.

[24] D. LeBlanc and R. Biddle, "Risk perception of internet-related activities," 2012 10th Annu. Int. Conf. Privacy, Secur. Trust. PST 2012, pp. 88-95, 2012, doi: 10.1109/PST.2012.6297924.

[25] A. R. Anggraini and J. Oliver, “済無No Title No Title,” J. Chem. Inf. Model., vol. 53, no. 9, pp. 1689-1699, 2019, doi: 10.1017/CBO9781107415324.004.

[26] E. R. Arboleda, "Secure and fast chaotic el gamal cryptosystem," Int. J. Eng. Adv. Technol., vol. 8, no. 5, pp. 1693-1699, 2019.

[27] G. Kalpana, P. V. Kumar, S. Aljawarneh, and R. V. Krishnaiah, "Shifted Adaption Homomorphism Encryption for Mobile and Cloud Learning," Comput. Electr. Eng., vol. 65, pp. 178-195, 2018, doi: 10.1016/j.compeleceng.2017.05.022.

[28] C. Gentry, "A Fully Homomorphic Encryption Scheme," Dissertation, no. September, p. 169, 2009, doi: $10.1145 / 1536414.1536440$

[29] A. B. Alexandru, K. Gatsis, Y. Shoukry, S. A. Seshia, P. Tabuada, and G. J. Pappas, "Cloud-based Quadratic Optimization with Partially Homomorphic Encryption," vol. 9286, no. c, pp. 1-8, 2018, doi: $10.1109 /$ tac.2020.3005920

[30] L. Xiong, D. Dong, Z. Xia, and X. Chen, "High-Capacity Reversible Data Hiding for Encrypted Multimedia Data with Somewhat Homomorphic Encryption," IEEE Access, vol. 6, pp. 60635-60644, 2018, doi: 10.1109/ACCESS.2018.2876036

[31] P. Mell and T. Grance, "The NIST definition of cloud computing. National Institute of Standards and Technology. Special Publication 800-145. 2011.” 2019. 